\title{
Enhancing rare variant interpretation in inherited arrhythmias through quantitative analysis of consortium disease cohorts and population controls
}

\author{
A full list of authors and affiliations appears at the end of the paper.
}

\begin{abstract}
Purpose: Stringent variant interpretation guidelines can lead to high rates of variants of uncertain significance (VUS) for genetically heterogeneous disease like long QT syndrome (LQTS) and Brugada syndrome (BrS). Quantitative and disease-specific customization of American College of Medical Genetics and Genomics/Association for Molecular Pathology (ACMG/AMP) guidelines can address this false negative rate.
\end{abstract}

Methods: We compared rare variant frequencies from 1847 LQTS (KCNQ1/KCNH2/SCN5A) and $3335 \mathrm{BrS}$ (SCN5A) cases from the International LQTS/BrS Genetics Consortia to population-specific gnomAD data and developed disease-specific criteria for ACMG/ AMP evidence classes-rarity (PM2/BS1 rules) and case enrichment of individual (PS4) and domain-specific (PM1) variants.

Results: Rare SCN5A variant prevalence differed between European (20.8\%) and Japanese (8.9\%) BrS patients ( $p=$ $\left.5.7 \times 10^{-18}\right)$ and diagnosis with spontaneous $(28.7 \%)$ versus induced (15.8\%) Brugada type 1 electrocardiogram (ECG) $(p=$ $\left.1.3 \times 10^{-13}\right)$. Ion channel transmembrane regions and specific $\mathrm{N}$ terminus $(\mathrm{KCNH} 2)$ and $\mathrm{C}$-terminus $(\mathrm{KCNQ} 1 / \mathrm{KCNH} 2)$ domains were characterized by high enrichment of case variants and $>95 \%$ probability of pathogenicity. Applying the customized rules, $17.4 \%$ of European BrS and $74.8 \%$ of European LQTS cases had (likely) pathogenic variants, compared with estimated diagnostic yields (case excess over gnomAD) of $19.2 \% / 82.1 \%$, reducing VUS prevalence to close to background rare variant frequency.

Conclusion: Large case-control data sets enable quantitative implementation of ACMG/AMP guidelines and increased sensitivity for inherited arrhythmia genetic testing.

Genetics in Medicine (2021) 23:47-58; https://doi.org/10.1038/s41436020-00946-5

Keywords: variant interpretation; LQTS; Brugada; ACMG/AMP guidelines

\section{INTRODUCTION}

The accurate interpretation of genetic variants remains one of the key challenges in clinical genetic testing for inherited cardiac conditions. It carries major clinical implications due to both the danger of assigning causality to variants that are in fact benign (false positive findings) as well as the possibility that variants that are truly causal are considered as variants of uncertain significance. The relatively high rate of rare benign variants in many genes (revealed by population sequencing studies such as gnomAD), and the subsequent debunking of many gene and variant associations with disease in the scientific literature, ${ }^{1,2}$ have highlighted the extent of this challenge and the danger of false positive classifications.

Variant interpretation guidelines produced by the American College of Medical Genetics and Genomics and the Association for Molecular Pathology (ACMG/AMP) in 2015 have brought much needed consistency and stringency to variant classification in both clinical and research laboratories. ${ }^{3}$ However, standard implementation of such guidelines may lead to overly conservative interpretation and a substantial rate of variants of uncertain significance (VUS) in clinical genetic testing. ${ }^{4}$ Interpretation is particularly challenging for nontruncating variants, i.e., those causing minor alterations in the protein sequence while preserving the reading frame (the majority of which are missense variants) whose functional consequences are harder to predict. For diseases with high allelic heterogeneity (i.e., thousands of potential diseasecausing variants) and where nontruncating variants are the predominant pathogenic variant class, this can correspond to a high rate of false negative results, evidenced when the frequency of VUS in cases exceeds the background rare variant frequency. ${ }^{1}$

Brugada syndrome (BrS) and long QT syndrome (LQTS) are inherited disorders that can cause severe arrhythmogenic events and sudden cardiac death in young individuals. Pathogenic variants in three genes account for the vast majority of genotype-positive cases for these conditions. These three genes code for $\alpha$-subunits of voltage-gated ion channels expressed in cardiomyocytes: SCN5A underlying the cardiac depolarizing sodium current $\left(I_{\mathrm{Na}}\right)$, and $\mathrm{KCNH} 2$ and KCNQ1 underlying the rapid $\left(I_{\mathrm{Kr}}\right)$ and slow $\left(I_{\mathrm{Ks}}\right)$ components of the repolarizing potassium delayed rectifier current. Rare

Correspondence: Roddy Walsh (r.t.walsh@amsterdamumc.nl)

A list of authors and their affiliations appears at the end of the paper

Submitted 1 June 2020; revised 3 August 2020; accepted: 11 August 2020

Published online: 7 September 2020 


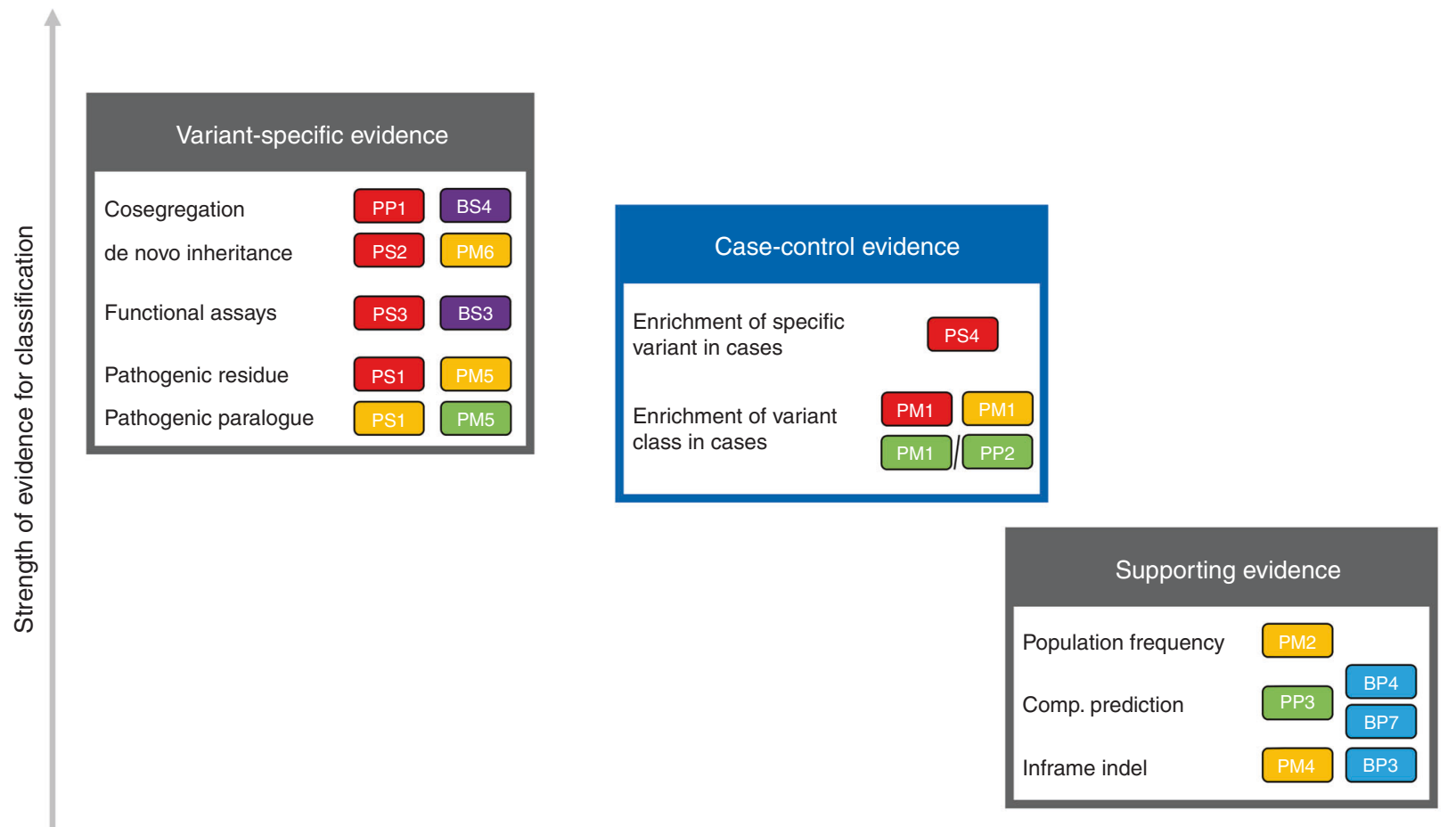

Availability of data

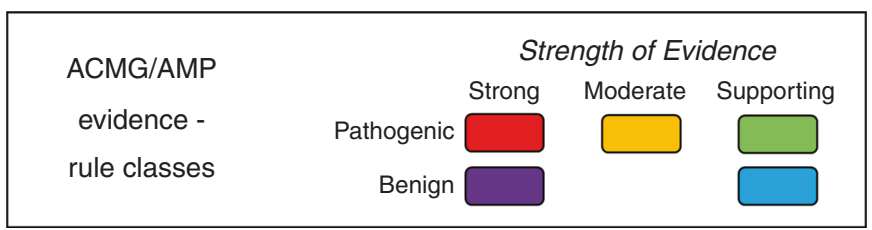

Fig. 1 For rare nontruncating variants in autosomal dominant disease, evidence classes and American College of Medical Genetics and Genomics/Association for Molecular Pathology (ACMG/AMP) rules (rule codes from Richards et al. ${ }^{3}$ ) can be broadly grouped by their power to distinguish between pathogenic and benign variants ( $y$-axis) and the likelihood that such evidence will be available ( $x$-axis). Variant-specific evidence (such as cosegregation in family pedigrees) is powerful but often unavailable for genetically heterogeneous diseases. Supporting evidence (such as population frequency) can be applied to most variants but is rarely sufficient for definitive classification. If available, data from case-control studies, relating to enrichment of specific variants or classes of variants, provide powerful gene/disease-specific evidence and help to address the high false negative rate associated with stringent contemporary guidelines.

coding variants in SCN5A cause BrS by a loss-of-function mechanism while LQTS is caused by functional loss-offunction variants in KCNQ1 (LQT1) and KCNH2 (LQT2) or gain-of-function variants in SCN5A (LQT3).

Genetic testing is well established for BrS and LQTS, to inform clinical management and facilitate cascade screening in families to identify at-risk carriers. ${ }^{5}$ Despite this, variant interpretation remains challenging for nontruncating variants that comprise the majority of causative variants in all three genes. For example, a recent reappraisal of all $S C N 5 A$ variants implicated in BrS using contemporary ACMG/AMP guidelines found that only $17 \%$ of missense variants could be classified as (likely) pathogenic. ${ }^{6}$ While this may partly reflect some erroneous disease associations in earlier reports, it highlights the difficulty in identifying pathogenic missense variants with these guidelines.

The classes of evidence used for variant interpretation can be broadly grouped based on their interpretative power and the frequency with which they can be applied (Fig. 1). For nontruncating variants not common in the population, supporting evidence, such as variant frequency in the population and computational predictions of variant effect, can be universally applied but has limited ability to distinguish between pathogenic and benign rare variation. These require informative variant-specific evidence to establish pathogenicity. However, due to the high allelic heterogeneity of these disorders, small pedigree sizes that preclude conclusive cosegregation analysis, and limited accessibility of functional assays, a large proportion of likely causative variants detected in patients with LQTS and $\mathrm{BrS}$ are unable to be classified as pathogenic. To address this false negative rate and improve the detection of pathogenic nontruncating variants, a third evidence category specific to gene-disease dyads can be applied: case-control studies that identify specific variants or classes of variants highly enriched in disease. Several disease and gene-specific implementations of the ACMG/AMP guidelines have been proposed by ClinGen 
and independent studies detailing how the rules, including those related to variant enrichment in cases over controls, should be applied for the particular genetic characteristics of each disease (Table S1). ${ }^{7}$

We have previously described a quantitative implementation of variant class enrichment evidence for sarcomeric genes involved in hypertrophic cardiomyopathy (HCM). ${ }^{4}$ By identifying gene regions highly enriched for rare variants in HCM cohorts compared with population controls, we were able to substantially decrease the rate of VUS in HCM cases using adapted ACMG/AMP guidelines (14-20\% increase in actionable HCM variants). Particular ion channel domains are also known to be enriched for cases variants, as previously demonstrated for LQTS $^{8,9}$ and BrS. ${ }^{10}$ Here, using large case cohorts from international consortia (1847 LQTS and $3335 \mathrm{BrS}$ cases), we apply and refine our ACMG/AMP guidelines adaptation to the three major disease genes of $\mathrm{BrS}$ and LQTS. We demonstrate how we can identify and classify high confidence pathogenic variant classes in specific clinical genetics contexts, and apply this evidence within the ACMG/AMP framework to enable an improved balance between sensitivity and specificity in clinical genetic testing for these conditions.

\section{Ethics statement}

\section{MATERIALS AND METHODS}

All subjects or their guardians provided informed consent and the study was approved by local ethical review committees of the participating centers (see Supplemental Methods for the list of committees).

\section{Patient and control cohorts}

The primary arrhythmia cohorts comprised patients diagnosed with BrS or LQTS recruited by the International BrS Genetics Consortium and the International LQTS Genetics Consortium, respectively. ${ }^{5,11}$ The BrS cohort consisted of 2400 unrelated cases of European origin from 23 predominantly European referral centers and 935 unrelated cases from 5 Japanese centers. The LQTS cohort consisted of 1394 unrelated cases of European origin and 453 unrelated cases from Japan (Table S2). All ECGs were centrally assessed by a genetic cardiac electrophysiologist prior to inclusion. For European cases, genetic ancestry was confirmed using genotypic principal component analysis. All subjects or their guardians provided informed consent and the study was approved by local ethical review committees of the participating centers.

Rare variant data from diagnostic sequencing of SCN5A (BrS) and KCNQ1, KCNH2, and SCN5A (LQTS) were provided by the different centers and curated based on the following canonical transcripts: ENST00000155840 (KCNQ1), ENST00000262186 (KCNH2) and ENST00000333535 (SCN5A). Details of all rare variants are provided in Tables S3/S4. Three additional arrhythmia-related data sets detailed in Supplemental Methods and Table S2 were used for further analyses as described in Results.

The gnomAD data set (version 2.1) comprising 125,748 individuals with exome sequencing was used for population controls, with the following population-specific subsets used for the European and Japanese cases respectively: non-Finnish European (gnomAD-NFE) comprising 56,885 individuals and East Asian (gnomAD-EAS) comprising 9197 individuals.

Defining population frequency thresholds for rare variants The maximum credible population allele frequency thresholds for rare, pathogenic variants were calculated as previously described: ${ }^{12}$

\section{prevalence of disease $\times$ maximum allelic contribution \\ penetrance}

where the maximum allelic contribution is the maximum proportion of cases potentially attributable to a single causative variant in these cohorts.

Population frequency thresholds were calculated on the basis of two estimates of penetrance-50\% and 10\%-for both $\mathrm{BrS}$ and LQTS. For BrS, we used a disease prevalence of 1/ 1000 and a maximum allelic contribution of 0.01 (based on SCN5A:c.4813+5_4813+6insGTGG, attributable to $15 / 2400$ or $0.6 \%(0.3-1.0 \%)$ of cases in this study). The thresholds for $\mathrm{BrS}$ were calculated as $1.0 \times 10^{-5}$ and $5.0 \times 10^{-5}$ respectively. For LQTS, we used a disease prevalence of $1 / 2000$ and maximum allelic contribution of 0.022 (based on KCNQ1: c. $1032 \mathrm{G}>\mathrm{A}$, attributable to $29 / 1847$ or $1.6 \%(1.1-2.2 \%)$ of cases in this study). The thresholds for LQTS were calculated as $1.1 \times 10^{-5}$ and $5.5 \times 10^{-5}$ respectively.

\section{Defining case-enriched regions in arrhythmia genes}

To define the relative enrichment of rare variants in cases over controls for arrhythmia gene regions, we used the etiological fraction (EF), which in the context of Mendelian disease estimates the proportion of cases with a rare variant of a particular class in whom that variant is disease-causing. The $\mathrm{EF}$ is derived from the odds ratio (OR) by the formula: $\mathrm{EF}=$ $(\mathrm{OR}-1) / \mathrm{OR}$, with $95 \%$ confidence intervals (CI) calculated as described in Supplemental Methods. For the three arrhythmia genes (KCNQ1/KCNH2/SCN5A), EFs were calculated for rare nontruncating (missense and inframe insertions/deletions) variants by comparing arrhythmia cohorts with populationrelevant gnomAD controls. EFs were calculated for specific gene/protein regions in $K C N Q 1 / K C N H 2 / S C N 5 A$, defined according to recognized domains in the respective UniProt (version 207) entries (details in Results and Supplemental Methods), as well as the KCNQ1 C-terminus highly conserved regions defined by Kapplinger at al. ${ }^{9}$ and the KCNH2 Nterminus cluster based on distribution of LQTS variants in this study. Regions with poor coverage in gnomAD and founder/recurrent case variants that may inflate regional EFs were masked from this analysis (see details in Supplemental Methods).

\section{Variant interpretation with standard ACMG/AMP guidelines}

Case variants were classified according to ACMG/AMP guidelines using the CardioClassifier application ${ }^{13}$ with adaptations 
for the following rules. First, variant population frequency (PM2/BS1 rules) used gnomAD exomes filtering allele frequencies (FAF) with thresholds defined in this study. Second, loss-offunction evidence (PVS1) was implemented according to recommendations from the ClinGen Sequence Variant Interpretation Workgroup. ${ }^{14}$ Third, functional evidence rules (PS3/ BS3) for BrS/SCN5A were applied using published data curated by Denham et al. ${ }^{6}$ and a high-throughput functional study by Glazer et al. ${ }^{15}$ Note that evidence relating to cosegregation (PP1/ BS4), de novo inheritance (PS2/PM6), and functional data for LQTS (PS3/BS3) could not be applied due to the lack of curated resources. While this will affect the yield of (likely) pathogenic variants, cosegregation evidence is not expected to be particularly informative for BrS as family pedigrees tend to be of limited sizes with numerous examples of non-Mendelian segregation. 6,16

\section{Variant interpretation with case-control evidence}

We then implemented refined ACMG/AMP guidelines based on the case-control evidence we generated to assess the effect on the yield of (likely) pathogenic variants. For the enrichment of classes of rare nontruncating variants (PM1 rule), EF data from the protein region analysis were applied. $P M 1$ strong was applied for rare variants in regions with an $\mathrm{EF} \geq 0.95$ (equivalent to $\mathrm{OR} \geq 20$ ), $P M 1$ 1_moderate where $0.95>\mathrm{EF} \geq 0.90 \quad(20>\mathrm{OR} \geq 10)$, and $P M 1 \_$supporting where $0.90>\mathrm{EF} \geq 0.80 \quad(10>\mathrm{OR} \geq 5)$, as previously described. ${ }^{4}$ The EF threshold of 0.95 implies a $95 \%$ or greater prior probability of pathogenicity and therefore the likely pathogenic classification for variants with $\mathrm{EF} \geq 0.95$ (PM1_strong, PM2) aligns with variant interpretation guidelines. ${ }^{3}$ Individual variants significantly enriched in cases over populationspecific gnomAD controls were identified (minimum case count of 3 and Fisher's exact $p$ value $<0.05$ with Bonferroni multiple testing correction for the number of case variants assessed for each condition). The PS4 rule strength was applied based on the lower boundary of the $95 \%$ CI of the OR, $\geq 20, \geq 10$, and $\geq 5$ for strong, moderate, and supporting evidence respectively (equivalent to the PM1 OR thresholds). See Supplemental Methods for full details of how each ACMG/AMP rule was applied.

\section{RESULTS}

Defining population frequency thresholds for rare variants To define appropriate population allele frequency thresholds for $\mathrm{BrS}$ and LQTS (i.e., the frequency above which variants are unlikely to be causative), we computed disease odds ratios (OR) across different frequency bins (comparing nontruncating variants from European arrhythmia case cohorts and gnomAD-NFE population controls). Disease-specific frequency thresholds were calculated as described in Materials and Methods for variant penetrance estimates of $10 \%$ and $50 \%$ $\left(5.0 \times 10^{-5}\right.$ and $1.0 \times 10^{-5}$ for BrS, $5.5 \times 10^{-5}$ and $1.1 \times 10^{-5}$ for LQTS respectively), which were used as upper frequency thresholds along with $1 \times 10^{-3}$ (BA1 benign variant threshold ${ }^{13}$ ) (Fig. 2a and Table S5).

Variants in the second rarest bin, i.e., corresponding to estimated penetrance between $10 \%$ and $50 \%$, were either not
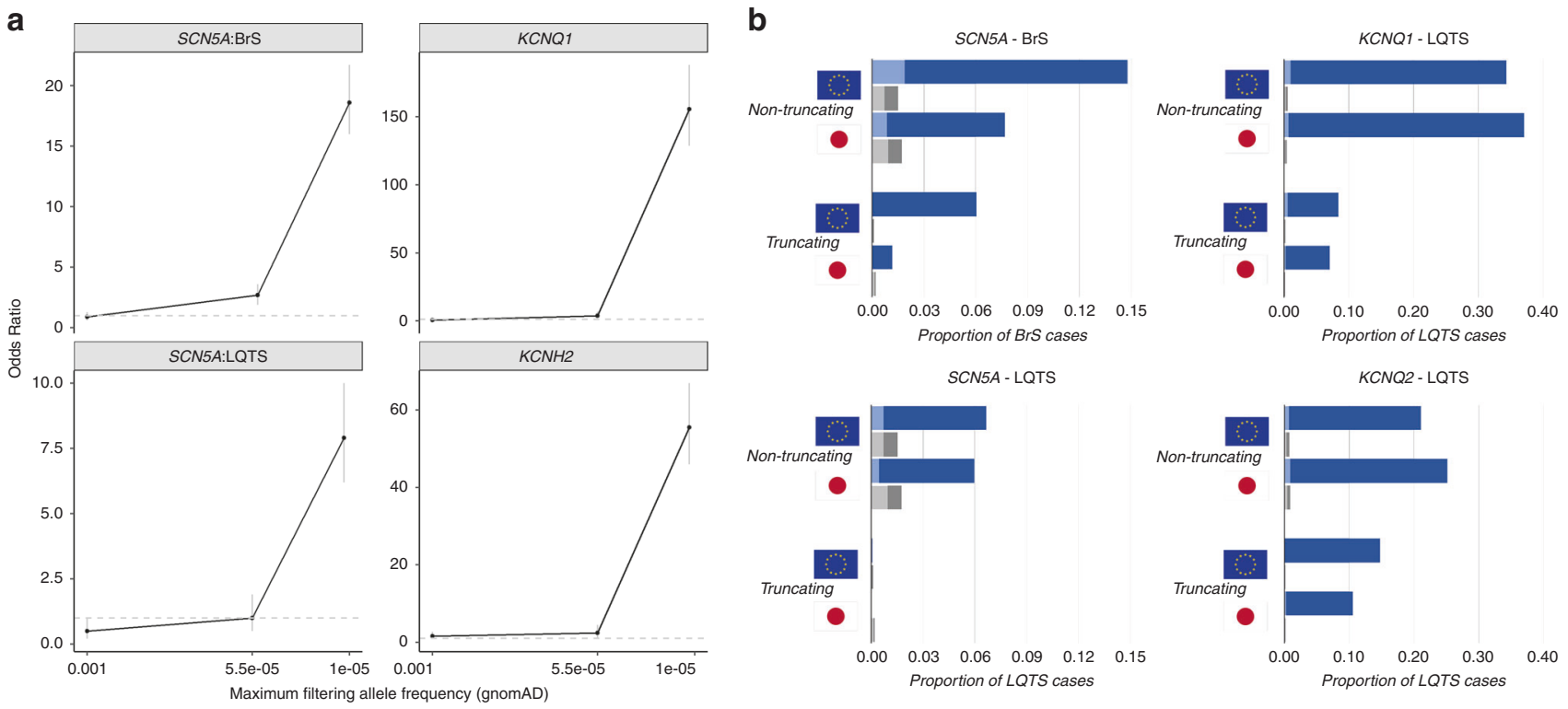

Fig. 2 Frequency of rare variants in $K C N Q 1, K C N H 2$ and SCN5A in inherited arrhythmia cohorts and gnomAD population controls. (a) The odds ratio for disease association for long QT syndrome (LQTS) (KCNQ1, KCNH2, SCN5A) and Brugada syndrome (BrS) (SCN5A) stratified by filtering allele frequency, based on the prevalence of rare variants in the European arrhythmia cohorts and gnomAD exomes. Data for each bin are plotted at the upper frequency cutoff. Error bars represent 95\% confidence intervals (Cls). The dashed gray line indicates an odds ratio (OR) of 1. (b) Proportion of cases in the $\mathrm{BrS}$ and LQTS European and Japanese cohorts with rare nontruncating (missense and inframe insertions/deletions) and truncating (frameshift, nonsense, splice) variants (blue) and comparison with the frequency of such variants in population-specific gnomAD data sets (gray). The darker shades indicate the rarest variants corresponding to an estimated penetrance of $\geq 50 \%$ filtering allele frequencies (FAF $<1.0 \times 10^{-5}$ and $1.1 \times 10^{-5}$ for BrS and LQTS respectively), while the lighter shades represent variants in the $10-50 \%$ penetrance range. 
significantly enriched in cases or had substantially lower ORs compared with the rarest bin (where penetrance $\geq 50 \%): \quad 10-50 \%: 3.8 \quad(2.2-6.6)$ and $>50 \%: 155.8$ (128.9-188.2) for KCNQ1/LQTS, 10-50\%:2.4 (1.2-4.5) and $>50 \%: 55.5 \quad(46.0-67.0)$ for KCNH2/LQTS, $10-50 \%: 1.0$ $(0.5-1.9)$ and $>50 \%: 7.9(6.2-10.0)$ for SCN5A/LQTS, and 10-50\%:2.7 (1.9-3.6) and >50\%:18.6 (16.0-21.7) for SCN5A/BrS. No significant enrichment of variants with frequencies between $1 \times 10^{-3}$ and $5 \times 10^{-5} / 5.5 \times 10^{-5}$ was observed for any gene.

These results indicate that while some variants with a population frequency above the more stringent thresholds may be disease-causing, the lower signal-to-noise ratio and presumably lower penetrance of such variants suggest that a higher burden of proof should be required to classify them as (likely) pathogenic (e.g., demonstration of functional effects or statistical enrichment in cases). For subsequent analysis in this study, we used the higher, more inclusive thresholds (based on estimated penetrance of $10 \%$ ) for describing the prevalence of rare variants in arrhythmia cohorts. However, the more stringent thresholds (based on estimated penetrance of $\geq 50 \%$ ) were used when applying the PM2/PM1 variant classification rules to minimize false positive classifications. The PM2 rule (rarity in population) was therefore applied to variants with a gnomAD exomes $\mathrm{FAF}<1.0 \times 10^{-5}(\mathrm{BrS})$ and $1.1 \times 10^{-5}$ (LQTS) and the BS1 rule (frequency too high for the disorder) was applied to variants with a FAF $>5.0 \times 10^{-5}(\mathrm{BrS})$ and $5.5 \times 10^{-5}$ (LQTS), with a recommended "gray zone" ${ }^{\text {"7,17 }}$ where neither is applied for the potential low penetrance variants.

\section{Prevalence of rare variants in primary arrhythmia cohorts}

The prevalence of rare $\left(\mathrm{FAF}<5 \times 10^{-5}\right)$ SCN5A variants in the $\mathrm{BrS}$ cohort was significantly different between the European and Japanese cohorts, detected in $20.8 \%$ and $8.9 \%$ of cases respectively $\left(p=5.7 \times 10^{-18}\right)$ (Fig. $2 \mathbf{b}$, Table S6). Nontruncating variants accounted for $71.0 \%$ and $86.7 \%$ of rare variants detected in the European and Japanese BrS cases respectively. The prevalence of rare $\left(\mathrm{FAF}<5.5 \times 10^{-5}\right)$ variants in KCNQ1, $\mathrm{KCNH} 2$, and SCN5A in the LQTS cohort was high (as expected) and similar between European and Japanese cohorts, detected in $85.4 \%$ and $85.9 \%$ of cases respectively $(p=0.63)$. The relative proportions of truncating and nontruncating variants in $K C N Q 1$ and $K C N H 2$ however are marginally different between the two populations $(p=0.007)$ (Fig. $2 \mathbf{b})$.

\section{Assessing enrichment of rare variants in cases in arrhythmia gene regions}

We next assessed variant distribution to identify gene/protein regions enriched for rare (corresponding to an estimated penetrance of $\geq 50 \%$ as described above) nontruncating variants in cases compared with gnomAD (Fig. 3a), as defined by the EF.

For BrS and LQTS, EFs were calculated separately by population, with European cases versus gnomAD-NFE and Japanese cases versus gnomAD-EAS (Tables 1, 2). For BrS, the yield of rare SCN5A variants was significantly higher for cases diagnosed with a spontaneous compared with a drug-induced type 1 electrocardiogram (ECG) pattern ( $28.7 \%$ vs. $15.8 \%$ in the European cases, $p=1.3 \times 10^{-13}$ ), therefore $\mathrm{EFs}$ were also calculated for European cases subclassified by ECG pattern (Table 1). For LQTS, as clinical genetic tests are often referred based on suspected rather than confirmed diagnosis, we also calculated EFs from a published referral cohort of 2500 cases, ${ }^{18}$ where the yield of rare variants in the three LQTS genes (33.6\%) reflects a lower proportion of definitive cases (Table 2).

Variants were highly enriched in cases in the transmembrane regions of all three genes (with the exception of SCN5A variants in LQTS cases) and select N/C-terminus domains in KCNQ1 and KCNH2 (Tables 1, 2). The regional EFs produced by this analysis, and consequently the strength of the evidence applied (PM1 rule), display marked variability by ancestry (BrS), diagnostic certainty (LQTS), and diagnostic criteria (BrS spontaneous/drug-induced type 1 ECG pattern), highlighting the importance of considering these contexts for variant interpretation.

\section{Identification of specific variants enriched in arrhythmia case cohorts}

Based on European and Japanese case-control comparisons as described in Materials and Methods, 48 individual variants are enriched in $\mathrm{BrS}$ cases (with 2, 26, and 20 variants activating the PS4 strong, moderate, and supporting rules respectively) (Fig. S1, Table S9) and 108 variants are enriched in LQTS cases (with 43,54, and 11 variants activating the PS4 strong, moderate, and supporting rules respectively) (Fig. S2, Table S10).

\section{ACMG/AMP classification of rare variants in arrhythmia cohorts}

We classified the variants in the BrS and LQTS cohorts according to the ACMG/AMP guidelines, using the CardioClassifier application ${ }^{13}$ (with adaptations as described in Materials and Methods). The frequency of rare variants in gnomAD estimates the rate of rare benign variants in both population and arrhythmia cohorts, and can be used to assess the sensitivity (and specificity) of variant classification approaches. In particular, the excess of VUS over this gnomAD frequency denotes the false negative rate in clinical genetic testing, i.e., the proportion of cases with variants that are expected to be disease-causing but for which insufficient evidence currently exists to classify them as (likely) pathogenic.

For BrS, classification was performed before and after inclusion of the case-control evidence described above (PM1/ PS4 rules), to assess the effect of these approaches on the diagnostic yield. With the basic ACMG/AMP rules (including functional evidence but excluding segregation data), 9.1\% of European BrS cases had a rare nontruncating SCN5A VUS compared with $1.5 \%$ in gnomAD, highlighting a large false negative rate. After applying the case-control PM1/PS4 evidence, the proportion of cases with VUS was reduced to 3.1\% (Fig. 3b). Given the more modest EFs associated with variants found solely in Japanese BrS cases, the VUS rate was 
a

KCNH2

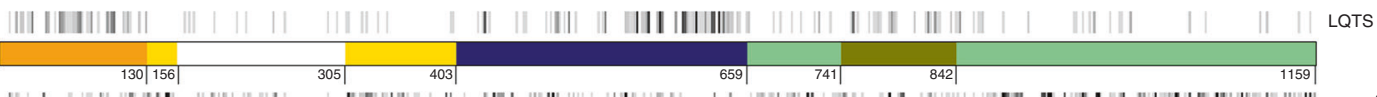

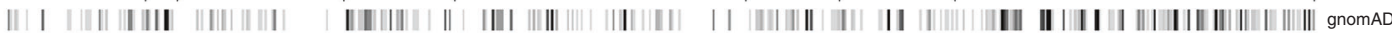

SCN5A

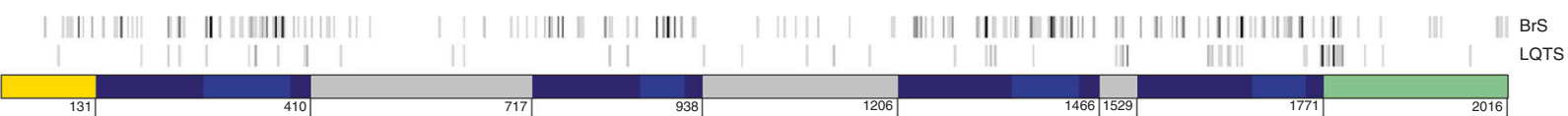

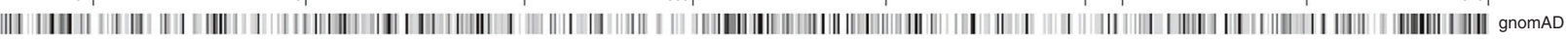

Domains: $\square$ N-terminus $\square$ N-terminus cluster $\square$ Transmembrane/Linker/Pore $\square$ TM pore segment $\square$ Interdomain Linke

C-terminus C-terminus highly conserved regions $\square$ Cyclic nucleotide binding domain (cNBD) $\square$ Masked
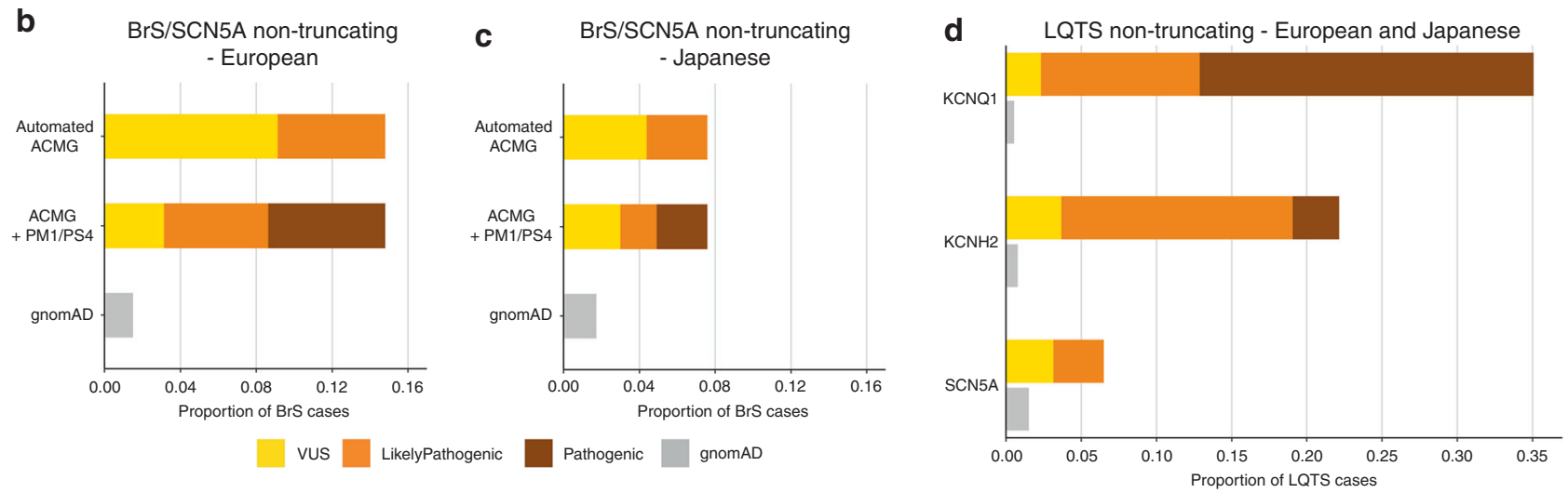

Fig. 3 Identification of ion channel gene domains enriched in case variants and effect of case-control analyses on variant classification in inherited arrhythmia patients. (a) Distribution of rare, nontruncating variants in the primary Brugada syndrome (BrS) and long QT syndrome (LQTS) cohorts across the domains of KCNQ1, KCNH2, and SCN5A and equivalent variant classes in gnomAD (full data set). Domain coordinates are derived from UniProt entries, with the exception of the KCNQ1 C-terminus highly conserved regions (from Kapplinger et al. ${ }^{9}$ ) and the $K C N H 2 \mathrm{~N}$-terminus cluster (based on variant distribution observed in this cohort and published referral cohort $\left.{ }^{18}\right)$. Regions with poor coverage in gnomAD exome sequencing, and therefore excluded from etiological fraction (EF) calculations, are in white. Darker gray indicates higher variant density (overlapping variants not plotted separately). The coordinates describe amino acid position. (b-d) Effect of case-control evidence (PM1/PS4 rules) on American College of Medical Genetics and Genomics/Association for Molecular Pathology (ACMG/AMP) classification of rare nontruncating variants. For BrS, the proportion of cases with pathogenic, likely pathogenic, and variants of uncertain significance (VUS) are displayed before and after use of these evidence classes, for European (b) and Japanese (c) cases. Classification using case-control evidence for both European and Japanese LQTS cases is shown in (d). The sensitivity of variant classification methods can be measured by comparison with the rate of rare benign variation in gnomAD (gray); any excess beyond this is expected to reflect pathogenic variation in cases and therefore represents the target cohort yield of pathogenic variants.

reduced from $4.4 \%$ to $3.0 \%$ in Japanese BrS patients (compared with a $1.7 \%$ background rate in gnomAD-EAS) (Fig. $3 c$ ).

For LQTS, familial cosegregation and functional evidence are critical for the classification of nontruncating variants (all were VUS with the basic ACMG/AMP classification) but given the extensive allelic heterogeneity (504 distinct rare nontruncating variants in 1847 cases) and the lack of highthroughput functional studies or curated resources, it was not feasible to include this evidence here. However, using the case-control PM1/PS4 evidence, the proportion of cases with VUS was reduced to $2.33 \%$ for KCNQ1 (compared with a gnomAD background frequency of $0.53 \%$ ), $3.68 \%$ for $\mathrm{KCNH} 2$ (gnomAD $=0.77 \%$ ), and $3.14 \%$ for SCN5A (gnomAD $=$ 1.5\%) (Fig. 3d).
Of the variants upgraded from VUS to (likely) pathogenic, only $11.1 \%$ of $\mathrm{BrS}$ and $36.1 \%$ of LQTS variants had a nonconflicting pathogenic or likely pathogenic classification in ClinVar (Tables S11/S12).

\section{Reclassification of published BrS SCN5A variants}

We also reanalyzed the set of published BrS-implicated SCN5A variants that were classified with contemporary ACMG/AMP guidelines by Denham et al., ${ }^{6}$ where $72 \%$ (231/321) of nontruncating SCN5A variants were classified as VUS. Applying the case-control (PS4 and PM1 based on all European BrS cases) and frequency (PM2/BS1) evidence described here, 152 of these VUS (65.8\%) were upgraded to (likely) pathogenic (Table S13). 
Table 1 Etiological fraction (EF) values for gene regions/domains in SCN5A based on comparison of rare (filtering allele frequency $[F A F]<1 \times 10^{-5}$ ) nontruncating variants in Brugada syndrome $(\mathrm{BrS})$ and gnomAD population cohorts.

\begin{tabular}{|c|c|c|c|c|c|c|c|c|c|c|c|c|c|}
\hline \multirow[t]{2}{*}{ SCN5A Region/Domain } & \multirow[t]{2}{*}{ Amino acid residues } & \multicolumn{3}{|c|}{$\begin{array}{l}\text { European BrS spontaneous } \\
\text { Type } 1 \text { ECG vs gnomAD-NFE }\end{array}$} & \multicolumn{3}{|c|}{$\begin{array}{l}\text { European BrS induced Type } \\
1 \text { ECG vs gnomAD-NFE }\end{array}$} & \multicolumn{3}{|c|}{$\begin{array}{l}\text { European BrS all cases vs } \\
\text { gnomAD-NFE }\end{array}$} & \multicolumn{3}{|c|}{$\begin{array}{l}\text { Japanese BrS cases vs } \\
\text { gnomAD-EAS }\end{array}$} \\
\hline & & gnomAD & BrS & $\mathrm{EF}$ & gnomAD & BrS & EF & gnomAD & BrS & EF & gnomAD & BrS & EF \\
\hline $\begin{array}{l}\text { Transmembrane } \\
\text { regions }\end{array}$ & \begin{tabular}{|l|}
$132-410,718-938$ \\
$1207-1466,1530-1771$ \\
\end{tabular} & $0.31 \%$ & $14.67 \%$ & 0.982 & $0.31 \%$ & $7.78 \%$ & 0.964 & $0.31 \%$ & $10.58 \%$ & 0.974 & $0.37 \%$ & $4.28 \%$ & 0.918 \\
\hline $\begin{array}{l}\text { Transmembrane - } \\
\text { pore segments }\end{array}$ & \begin{tabular}{|l} 
273-389, 857-917, \\
1354-1444, 1675-1748
\end{tabular} & $0.09 \%$ & $8.22 \%$ & 0.989 & $0.09 \%$ & $3.89 \%$ & 0.977 & $0.09 \%$ & $5.58 \%$ & 0.984 & $0.17 \%$ & $2.14 \%$ & 0.924 \\
\hline $\begin{array}{l}\text { Transmembrane - } \\
\text { other regions }\end{array}$ & - & $0.21 \%$ & $6.44 \%$ & 0.969 & $0.21 \%$ & $3.89 \%$ & 0.947 & $0.21 \%$ & $5.00 \%$ & 0.959 & $0.20 \%$ & $2.14 \%$ & 0.908 \\
\hline $\mathrm{N}$-terminus & $1-131$ & $0.05 \%$ & $0.78 \%$ & 0.931 & $0.05 \%$ & $0.49 \%$ & 0.889 & $0.05 \%$ & $0.58 \%$ & 0.908 & $0.07 \%$ & $0.11 \%$ & 0.373 \\
\hline Interdomain Linkers & $\begin{array}{l}\text { 411-717, 939-1206, } \\
1467-1529\end{array}$ & $0.32 \%$ & $1.00 \%$ & 0.684 & $0.32 \%$ & $0.69 \%$ & 0.544 & $0.32 \%$ & $0.79 \%$ & 0.600 & $0.24 \%$ & $0.43 \%$ & 0.448 \\
\hline C-terminus & $1772-2016$ & $0.11 \%$ & $0.67 \%$ & 0.833 & $0.11 \%$ & $0.35 \%$ & 0.679 & $0.11 \%$ & $0.46 \%$ & 0.757 & $0.12 \%$ & $0.43 \%$ & 0.713 \\
\hline
\end{tabular}

EF values are colored according to the PM1 rule activated: strong (red), moderate (orange), supporting (green), and none (black). The comparisons shown are European $\mathrm{BrS}$ cases with a spontaneous type 1 electrocardiogram (ECG) $(n=900)$ vs. gnomAD-NFE, European BrS cases with an induced type 1 ECG $(n=1440)$ vs. gnomAD-NFE, all European BrS cases $(n=2400)$ vs. gnomAD-NFE and Japanese BrS cases $(n=935)$ vs. gnomAD-EAS (see Table S7 for full details). This evidence should be used only for nontruncating SCN5A variants detected in patients with BrS as follows: (1) check the variant is rare (gnomAD FAF $\left.<1 \times 10^{-5}\right)$, $(2)$ select the appropriate comparison depending on patient ethnicity (European ancestry or Japanese) and type 1 ECG pattern of the patient (spontaneous, induced or use "all cases" if unknown), (3) select the PM1 evidence level (based on EF) depending on the SCN5A region/domain where the variant is located.

\section{Application to sudden arrhythmic death syndrome}

As well as clinical genetic testing for individuals with BrS/ LQTS, sequencing of the major arrhythmia genes is also often performed for sudden arrhythmic death syndrome (SADS) cases, i.e., sudden death in usually nonelderly individuals with a structurally normal heart, including the coronary arteries. We assessed the enrichment of rare variants (FAF $<1 \times 10^{-5}$ ) in KCNQ1, KCNH2, SCN5A, and RYR2 in a cohort of 505 SADS cases compared with gnomAD to calculate EFs in the context of SADS and identify interpretable variant classes. ${ }^{19}$ The yield of rare nontruncating variants in the SADS cohort was low: KCNQ1 (1.4\%), KCNH2 (1.8\%), SCN5A (1.8\%), and RYR2 (5.0\%), with EFs for most protein regions either too low for PM1 rule application $(\mathrm{EF}<0.8)$ or based on very limited case variants. The exception are variants in the pathogenic hotspots of RYR2 identified in our previous study, ${ }^{4}$ although the EF (0.88) yields only PM1_supporting evidence (Table S14).

\section{DISCUSSION}

In this study we have shown that large cohorts of genetically characterized arrhythmia patients can be used to develop disease- and gene-specific quantitative applications of the ACMG/AMP framework and increase the sensitivity of genetic testing in these conditions. Through case-control analysis with gnom $A D$ population data, we defined appropriate arrhythmia-specific population frequency thresholds to define variant rarity and then developed rules for variant enrichment evidence-the association of individual variants with disease (PS4) and enrichment of region-specific variants in case cohorts (PM1). The latter allows us to distinguish between highly pathogenic nontruncating variant classes (with some approaching truncating variant ORs) that can be confidently classified as likely pathogenic even when further corroborating evidence is absent and those with lower probabilities that require additional evidence to inform pathogenicity. By incorporating this evidence into ACMG/ AMP guidelines, the prevalence of VUS in the three arrhythmia genes are reduced close to the background rare variant frequency (Fig. 3).

As the domain-based PM1 rule adaptations depend on the case-control signal-to-noise ratio, these should be applied taking into account factors that affect this ratio (see Tables 1 and 2 for how to apply this evidence). These include patient ethnicity due to differences in rare variant population frequencies and disease genetic architecture, such as observed here between European and Japanese BrS cases. Diagnostic criteria such as spontaneous or induced type 1 ECG pattern in $\mathrm{BrS}$ patients may also influence diagnostic yield and hence the interpretability of detected variants. It is also critical to account for phenotype uncertainty in the individual being tested. Here, we provide guidelines for LQTS in the context of either a definitive diagnosis of disease or referral for genetic testing based on suspicion of disease, and demonstrate that this approach has limited utility for SADS genetic testing, given the extremely heterogeneous etiology underlying these cases.

We applied this method to rare variants defined with stringent population frequency thresholds, based on the published framework ${ }^{12}$ and estimating penetrance of at least 50\%. Such ultrarare variants have the strongest case-control signals (Fig. 2a), allowing us to identify interpretable variants with high confidence and minimize the risk of false positive classifications. We also observed a modest case excess of variants with population frequencies corresponding to lower estimated penetrance (10-50\%). 
Table 2 Etiological fraction (EF) values for gene regions/domains in KCNQ1, KCNH2, and SCN5A based on comparison of rare (filtering allele frequency $[F A F]<1.1 \times 10^{-5}$ ) nontruncating variants in long QT syndrome (LQTS) and gnomAD population cohorts.

\begin{tabular}{|c|c|c|c|c|c|c|c|c|c|c|c|}
\hline \multirow[t]{2}{*}{ Gene } & \multirow[t]{2}{*}{ Region/Domain } & \multirow[t]{2}{*}{ Amino acid residues } & \multicolumn{3}{|c|}{$\begin{array}{c}\text { European LQTS cohort vs } \\
\text { gnomAD-NFE }\end{array}$} & \multicolumn{3}{|c|}{$\begin{array}{c}\text { Japanese LQTS cohort vs } \\
\text { gnomAD-EAS }\end{array}$} & \multicolumn{3}{|c|}{$\begin{array}{l}\text { Referral LQTS cohort vs } \\
\text { gnomAD-ALL }\end{array}$} \\
\hline & & & gnomAD & LQTS & EF & gnomAD & LQTS & EF & gnomAD & LQTS & EF \\
\hline \multirow{4}{*}{ KCNQ1 } & Transmembrane/Linker/Pore & $122-348$ & $0.10 \%$ & $22.31 \%$ & 0.997 & $0.11 \%$ & $26.05 \%$ & 0.997 & $0.10 \%$ & $7.68 \%$ & 0.988 \\
\hline & $\mathrm{N}$-terminus & 71-121 & $0.04 \%$ & $0.65 \%$ & 0.940 & $0.02 \%$ & $0.00 \%$ & - & $0.03 \%$ & $0.24 \%$ & 0.887 \\
\hline & $\begin{array}{l}\text { C-terminus: Highly conserved } \\
\text { regions }\end{array}$ & $349-391,509-575,585-607$ & $0.05 \%$ & $8.68 \%$ & 0.995 & $0.05 \%$ & $5.74 \%$ & 0.992 & $0.05 \%$ & $3.24 \%$ & 0.986 \\
\hline & C-terminus: Other regions & $392-508,576-584,608-676$ & $0.11 \%$ & $0.86 \%$ & 0.879 & $0.07 \%$ & $0.88 \%$ & 0.923 & $0.10 \%$ & $0.44 \%$ & 0.765 \\
\hline & & & & & & & & & & & \\
\hline \multirow{5}{*}{$\mathrm{KCNH} 2$} & Transmembrane/Linker/Pore & 404-659 & $0.07 \%$ & $10.83 \%$ & 0.994 & $0.06 \%$ & $12.36 \%$ & 0.996 & $0.06 \%$ & $3.68 \%$ & 0.985 \\
\hline & $\mathrm{N}$-terminus: Cluster & $1-130$ & $0.03 \%$ & $4.38 \%$ & 0.994 & $0.02 \%$ & $5.74 \%$ & 0.996 & $0.03 \%$ & $1.72 \%$ & 0.984 \\
\hline & $\mathrm{N}$-terminus: Other regions & 131-156, 306-403 & $0.11 \%$ & $0.93 \%$ & 0.878 & $0.06 \%$ & $1.10 \%$ & 0.949 & $0.11 \%$ & $0.48 \%$ & 0.773 \\
\hline & C-terminus: cNBD & $742-842$ & $0.04 \%$ & $2.15 \%$ & 0.981 & $0.02 \%$ & $2.43 \%$ & 0.991 & $0.03 \%$ & $0.60 \%$ & 0.946 \\
\hline & C-terminus: Other regions & $660-741,843-1159$ & $0.20 \%$ & $2.08 \%$ & 0.906 & $0.24 \%$ & $2.65 \%$ & 0.913 & $0.20 \%$ & $0.80 \%$ & 0.751 \\
\hline & & & & & & & & & & & \\
\hline \multirow{4}{*}{ SCN5A } & Transmembrane/Linker/Pore & $\begin{array}{l}\text { 132-410, 718-938, 1207-1466, } \\
1530-1771\end{array}$ & $0.33 \%$ & $1.79 \%$ & 0.818 & $0.38 \%$ & $2.43 \%$ & 0.848 & $0.34 \%$ & $1.84 \%$ & 0.820 \\
\hline & $\mathrm{N}$-terminus & $1-131$ & $0.05 \%$ & $0.07 \%$ & 0.247 & $0.07 \%$ & $0.00 \%$ & - & $0.06 \%$ & $0.20 \%$ & 0.678 \\
\hline & Interdomain linkers & $411-717,939-1206,1467-1529$ & $0.30 \%$ & $0.65 \%$ & 0.540 & $0.24 \%$ & $0.88 \%$ & 0.734 & $0.28 \%$ & $1.00 \%$ & 0.725 \\
\hline & C-terminus & $1772-2016$ & $0.11 \%$ & $0.93 \%$ & 0.879 & $0.13 \%$ & $0.66 \%$ & 0.798 & $0.11 \%$ & $0.76 \%$ & 0.857 \\
\hline
\end{tabular}

EF values are colored according to the PM1 rule activated: strong (red), moderate (orange), supporting (green), and none (black). The comparisons shown are European LQTS cases ( $n=1394)$ vs. gnomAD-NFE, Japanese LQTS cases $(n=453)$ vs. gnomAD-EAS and the published LQTS referral cohort ( $n=2500)$ vs. gnomAD-ALL. It is uncertain whether the absence of KCNQ1 N-terminus variants in Japanese cases reflects a genuine difference between populations or is due to technical sequencing issues for this GC-rich region (see Table S8 for full details). This evidence should be used only for nontruncating KCNQ1, KCNH2, or SCN5A variants detected in patients with (or being genetically tested for) LQTS as follows: (1) check the variant is rare (gnomAD FAF $<1.1 \times 10^{-5}$ ); $(2)$ select the appropriate comparison-patients diagnosed with LQTS of European ancestry, patients diagnosed with LQTS of Japanese ancestry, or individuals of any ethnicity referred for LQTS genetic testing; (3) select the PM1 evidence level (based on EF) depending on the gene and region/domain where the variant is located.

Some of these variants, if detected in patients, may well contribute to the disease phenotype as genetic risk factors with modest effect sizes. However, given the much reduced signal-to-noise ratio, and the likelihood that many will be benign variants, a higher evidence threshold should be applied for establishing any pathogenic role. For this reason, we have not applied the PM1 or PM2 rules to such variants-more direct evidence such as enrichment of the specific variant in cases and validation with established functional assays is required.

Demonstrating significant enrichment of specific variants in disease cases is also potentially powerful evidence for pathogenicity when pedigree or functional data is not available. However, defining the significance of an observed enrichment and applying the appropriate evidence strength can be challenging. While most disease-specific ACMG/AMP framework implementations have proposed specific numbers of previously described cases for each evidence level (in the absence of large sequenced case cohorts), these can be arbitrary and do not demonstrate statistical enrichment (Table S1). The approach we describe here utilizes large multicenter arrhythmia cohorts to identify significantly enriched variants compared with population-matched gnomAD controls, adjusts for the disparity in cohort size between cases and controls, and applies conservative thresholds for the different evidence levels to minimize any false positive classifications. These methods can also easily incorporate any newly sequenced cohorts to identify additional enriched variants in the main arrhythmia genes. The specific PS4 variant evidence (detailed in Tables S9/S10) can be applied to all BrS/LQTS patients regardless of ethnicity or clinical context.

\section{Limitations}

The same primary arrhythmia cohorts were used to calculate regional EFs and then test the effect of PM1 evidence on variant classification. However, when accounting for population and disease diagnosis, we are confident that the observed variant frequencies will be consistent with other equivalent cohorts. We provide variant interpretation guidelines based on analysis of European and Japanese arrhythmia patients, therefore these should be used with caution for other ethnicities if there is an expectation of different case-control rare variant frequencies in other population groups. Large-scale sequencing of disease and population data sets for other populations groups remains a critical need, especially as quantitative classification methods like these are likely to be particularly beneficial for nonEuropean populations, given the increased difficulty of identifying pathogenic variants for such patients using standard guidelines. ${ }^{20}$ For LQTS we were unable to fully assess the additive effect of these methods on ACMG/AMP variant classification, as this is dependent on segregation and functional evidence for which comprehensive curated data sets are not available.

\section{Conclusion}

Through analysis of large sequenced case and control cohorts, we have developed quantitative and gene/disease-specific 
applications of ACMG/AMP variant interpretation guidelines for major arrhythmia genes that improve the balance between sensitivity and accuracy of clinical genetic testing and reduce the false negative rate associated with these genetically heterogeneous diseases. The findings also highlight that population and phenotype context are critical for effectively interpreting the clinical effect of rare variation. This study will have significant clinical impact in improving the diagnosis, treatment, and family screening of BrS/LQTS patients who currently receive uncertain genetic test results.

\section{SUPPLEMENTARY INFORMATION}

The online version of this article (https://doi.org/10.1038/s41436020-00946-5) contains supplementary material, which is available to authorized users.

\section{ACKNOWLEDGEMENTS}

R.W. is supported by an Amsterdam Cardiovascular Sciences fellowship. C.R.B. is supported by the Dutch Heart Foundation (CVON Predict-2/Concor-genes), Netherlands Organization for Scientific Research (VICI 016.150.610), and Fondation Leducq. See Supplement for all Acknowledgements.

\section{DISCLOSURE}

E.A. has received speaker fees from Biosense Webster. P.T.E. is supported by a grant from Bayer AG to the Broad Institute focused on the genetics and therapeutics of cardiovascular disease, and has consulted for Bayer AG, Novartis, and Quest Diagnostics. S.A.L. receives sponsored research support from Bristol Myers Squibb/Pfizer, Bayer AG, and Boehringer Ingelheim, and has consulted for Bristol Myers Squibb/Pfizer and Bayer AG. The other authors declare no conflicts of interest.

Publisher's note Springer Nature remains neutral with regard to jurisdictional claims in published maps and institutional affiliations.

\section{REFERENCES}

1. Walsh R, Thomson KL, Ware JS, et al. Reassessment of Mendelian gene pathogenicity using 7,855 cardiomyopathy cases and 60,706 reference samples. Genet Med. 2017;19:192-203.

2. Hosseini SM, Kim R, Udupa S, et al. Reappraisal of reported genes for sudden arrhythmic death. Circulation. 2018;138:1195-1205.

3. Richards S, Aziz N, Bale S, et al. Standards and guidelines for the interpretation of sequence variants: a joint consensus recommendation of the American College of Medical Genetics and Genomics and the Association for Molecular Pathology. Genet Med. 2015;17:405-423.

4. Walsh R, Mazzarotto F, Whiffin N, et al. Quantitative approaches to variant classification increase the yield and precision of genetic testing in Mendelian diseases: the case of hypertrophic cardiomyopathy. Genome Med. 2019;11:5.

5. Ackerman MJ, Priori SG, Willems $S$, et al. HRS/EHRA expert consensus statement on the state of genetic testing for the channelopathies and cardiomyopathies this document was developed as a partnership between the Heart Rhythm Society (HRS) and the
European Heart Rhythm Association (EHRA). Heart Rhythm. 2011;8:1308-1339.

6. Denham NC, Pearman CM, Ding WY, et al. Systematic re-evaluation of SCN5A variants associated with Brugada syndrome. J Cardiovasc Electrophysiol. 2019;30:118-127.

7. Harrison SM, Biesecker LG, Rehm HL. Overview of specifications to the ACMG/AMP variant interpretation guidelines. Curr Protoc Hum Genet. 2019;103:e93.

8. Kapa S, Tester DJ, Salisbury BA, et al. Genetic testing for long-QT syndrome: distinguishing pathogenic mutations from benign variants. Circulation. 2009;120:1752-1760.

9. Kapplinger JD, Tseng AS, Salisbury BA, et al. Enhancing the predictive power of mutations in the C-terminus of the KCNQ1-encoded Kv7.1 voltage-gated potassium channel. J Cardiovasc Transl Res. 2015;8:187-197.

10. Kapplinger JD, Giudicessi JR, Ye D, et al. Enhanced classification of Brugada syndrome-associated and long-QT syndrome-associated genetic variants in the SCN5A-encoded $\mathrm{Na}(\mathrm{V}) 1.5$ cardiac sodium channel. Circ Cardiovasc Genet. 2015;8:582-595.

11. Lahrouchi N, Tadros R, Crotti L, et al. Transethnic genome-wide association study provides insights in the genetic architecture and heritability of long QT syndrome. Circulation. 2020;142: 324-338.

12. Whiffin $N$, Minikel $E$, Walsh $R$, et al. Using high-resolution variant frequencies to empower clinical genome interpretation. Genet Med. 2017;19:1151-1158.

13. Whiffin N, Walsh R, Govind R, et al. CardioClassifier: disease- and genespecific computational decision support for clinical genome interpretation. Genet Med. 2018;20:1246-1254.

14. Abou Tayoun AN, Pesaran T, DiStefano MT, et al. Recommendations for interpreting the loss of function PVS1 ACMG/AMP variant criterion. Hum Mutat. 2018;39:1517-1524.

15. Glazer AM, Wada $Y$, Li B, et al. High-throughput reclassification of SCN5A variants. Am J Hum Genet. 2020;107:111-123.

16. Probst $V$, Wilde AAM, Barc J, et al. SCN5A mutations and the role of genetic background in the pathophysiology of Brugada syndrome. Circ Cardiovasc Genet. 2009;2:552-557.

17. Tavtigian SV, Greenblatt MS, Harrison SM, et al. Modeling the ACMG/ AMP variant classification guidelines as a Bayesian classification framework. Genet Med. 2018;20:1054-1060.

18. Kapplinger JD, Tester DJ, Salisbury BA, et al. Spectrum and prevalence of mutations from the first 2,500 consecutive unrelated patients referred for the FAMILION long QT syndrome genetic test. Heart Rhythm. 2009;6:1297-1303.

19. Lahrouchi N, Raju H, Lodder EM, et al. Utility of post-mortem genetic testing in cases of sudden arrhythmic death syndrome. J Am Coll Cardiol. 2017;69:2134-2145.

20. Landry LG, Rehm HL. Association of racial/ethnic categories with the ability of genetic tests to detect a cause of cardiomyopathy. JAMA Cardiol. 2018;3:341-345.

Open Access This article is licensed under a Creative Commons Attribution-NonCommercial-NoDerivatives 4.0 International License, which permits any non-commercial use, sharing, distribution and reproduction in any medium or format, as long as you give appropriate credit to the original author(s) and the source, and provide a link to the Creative Commons license. You do not have permission under this license to share adapted material derived from this article or parts of it. The images or other third party material in this article are included in the article's Creative Commons license, unless indicated otherwise in a credit line to the material. If material is not included in the article's Creative Commons license and your intended use is not permitted by statutory regulation or exceeds the permitted use, you will need to obtain permission directly from the copyright holder. To view a copy of this license, visit http://creativecommons.org/licenses/by-nc-nd/4.0/.

(C) The Author(s) 2020 
Roddy Walsh, PhD $\mathbb{1}^{1,2}$, Najim Lahrouchi, $\mathrm{MD}^{1,2}$, Rafik Tadros, MD, PhD ${ }^{3}$, Florence Kyndt, PharmD, PhD ${ }^{2,4}$, Charlotte Glinge, MD, PhD ${ }^{2,5}$, Pieter G. Postema, MD, PhD ${ }^{1,2}$, Ahmad S. Amin, MD, PhD ${ }^{1,2}$, Eline A. Nannenberg, MD, PhD ${ }^{2,6}$, James S. Ware, PhD, MRCP ${ }^{7,8,9}$, Nicola Whiffin, PhD 7,8,9, Francesco Mazzarotto, $\mathrm{PhD}^{7,8,10,11}$, Doris Škorić-Milosavljević, MD ${ }^{1,2}$, Christian Krijger, BSc ${ }^{1,2}$, Elena Arbelo, MD, PhD ${ }^{12,13,14}$, Dominique Babuty, MD, PhD ${ }^{15}$, Hector Barajas-Martinez, PhD ${ }^{16}$, Britt M. Beckmann, MD ${ }^{17}$, Stéphane Bézieau, PharmD, PhD ${ }^{2,4}$, J. Martijn Bos, MD, PhD ${ }^{18}$, Jeroen Breckpot, MD, PhD ${ }^{2,19}$, Oscar Campuzano, PhD ${ }^{14,20,21,22,}$ Silvia Castelletti, MD ${ }^{23}$, Candan Celen, $\mathrm{RN}^{24}$, Sebastian Clauss, MD ${ }^{17,25,26}$, Anniek Corveleyn, PhD ${ }^{2,27}$, Lia Crotti, MD, PhD 23,28,29,30, Federica Dagradi, MD ${ }^{23}$, Carlo de Asmundis, MD, PhD ${ }^{31}$, Isabelle Denjoy, $\mathrm{MD}^{2,32,33}$, Sven Dittmann, $\mathrm{PhD}^{2,34}$, Patrick T. Ellinor, $\mathrm{MD}, \mathrm{PhD}^{35,36}$, Cristina Gil Ortuño, MSc ${ }^{2,37}$, Carla Giustetto, MD ${ }^{38}$, Jean-Baptiste Gourraud, MD, 'PhD ${ }^{2,4}$, Daisuke Hazeki, MD, PhD ${ }^{39}$, Minoru Horie, MD, PhD ${ }^{40}$, Taisuke Ishikawa, DVM, PhD ${ }^{41}$, Hideki Itoh, MD, $\mathrm{PhD}^{42}$, Yoshiaki Kaneko, MD, $\mathrm{PhD}^{43}$, Jørgen K. Kanters, $\mathrm{MD}^{44}$, Hiroki Kimoto, MPharm ${ }^{45}$, Maria-Christina Kotta, MSC, PhD ${ }^{23,30}$, Ingrid P. C. Krapels, MD, PhD ${ }^{46}$, Masahiko Kurabayashi, MD, PhD ${ }^{43}$, Julieta Lazarte, $\mathrm{MSC}^{47}$, Antoine Leenhardt, MD ${ }^{2,32,33}$, Bart L. Loeys, MD, PhD ${ }^{48}$, Catarina Lundin, MD, PhD ${ }^{49}$, Takeru Makiyama, MD, PhD ${ }^{50}$, Jacques Mansourati, MD, PhD ${ }^{51}$, Raphaël P. Martins, MD, PhD ${ }^{52}$, Andrea Mazzanti, MD ${ }^{2,53}$, Stellan Mörner, MD, PhD ${ }^{2,54}$, Carlo Napolitano, MD, PhD 2,53 , Kimie Ohkubo, MD ${ }^{55}$, Michael Papadakis, MBBS, MD 2,56,57, Boris Rudic, MD ${ }^{58,59}$, Maria Sabater Molina, PhD 2,37 , Frédéric Sacher, MD, PhD ${ }^{60}$, Hatice Sahin, $\mathrm{RN}^{24}$, Georgia Sarquella-Brugada, MD, PhD ${ }^{2,21,61}$ Regina Sebastiano, BSc ${ }^{62}$, Sanjay Sharma, BSC, MD 2,56,57, Mary N. Sheppard, MD, FRCPath ${ }^{2,56,57}$ Keiko Shimamoto, $\mathrm{MD}^{63}$, M. Benjamin Shoemaker, MD ${ }^{64}$, Birgit Stallmeyer, $\mathrm{PhD}^{2,34}$, Johannes Steinfurt, MD ${ }^{65}$, Yuji Tanaka, MD, PhD ${ }^{66}$, David J. Tester, BS ${ }^{18}$, Keisuke Usuda, MD ${ }^{67}$, Paul A. van der Zwaag, MD, PhD ${ }^{68}$, Sonia Van Dooren, $\mathrm{PhD}^{2,69}$, Lut Van Laer, PhD ${ }^{48}$, Annika Winbo, MD, $\mathrm{PhD}^{70}$, Bo G. Winkel, $\mathrm{PhD}^{2,5}$, Kenichiro Yamagata, MD, $\mathrm{PhD}^{63}$, Sven Zumhagen, MD ${ }^{2,34}$, Paul G. A. Volders, MD, PhD ${ }^{71}$, Steven A. Lubitz, MD, MPH ${ }^{35,36}$, Charles Antzelevitch, PhD ${ }^{16}$, Pyotr G. Platonov, MD, PhD ${ }^{72}$, Katja E. Odening, MD ${ }^{65,73}$, Dan M. Roden, MD ${ }^{64,74,75}$, Jason D. Roberts, MD, MAS ${ }^{76}$, Jonathan R. Skinner, MB, ChB, MD ${ }^{77}$, Jacob Tfelt-Hansen, DMCs ${ }^{2,5,78}$, Maarten P. van den Berg, MD, PhD ${ }^{79}$, Morten S. Olesen, PhD ${ }^{80}$, Pier D. Lambiase, MD, PhD 2,81 , Martin Borggrefe, $\mathrm{MD}^{58,59}$, Kenshi Hayashi, MD, PhD ${ }^{67}$, Annika Rydberg, MD, PhD 2,82 , Tadashi Nakajima, MD, PhD ${ }^{43}$, Masao Yoshinaga, MD, PhD ${ }^{66}$, Johan B. Saenen, MD, PhD ${ }^{83}$, Stefan Kääb, MD, PhD ${ }^{17,25}$, Pedro Brugada, MD, PhD ${ }^{2,84}$, Tomas Robyns, MD, PhD ${ }^{2,85}$, Daniela F. Giachino, MD, PhD ${ }^{62,86}$, Michael J. Ackerman, $M D, P^{18}$, Ramon Brugada, MD, PhD ${ }^{87}$, Josep Brugada, MD, PhD ${ }^{88}$, Juan R. Gimeno, MD ${ }^{2,89}$, Can Hasdemir, MD ${ }^{24}$, Pascale Guicheney, $\mathrm{PhD}^{90}$, Silvia G. Priori, MD, PhD ${ }^{2,53}$, Eric Schulze-Bahr, MD, PhD 2,34, Naomasa Makita, MD, PhD ${ }^{41}$, Peter J. Schwartz, MD ${ }^{23,30,}$ Wataru Shimizu, MD, PhD ${ }^{91}$, Takeshi Aiba, MD, PhD ${ }^{63}$, Jean-Jacques Schott, PhD ${ }^{2,4}$, Richard Redon, $\mathrm{PhD}^{2,4}$, Seiko Ohno, MD, PhD ${ }^{92}$, Vincent Probst, MD, PhD ${ }^{2,4}$, Nantes Referral Center for inherited cardiac arrhythmia, Elijah R. Behr, MA, MD $2,56,57$, Julien Barc, PhD ${ }^{2,104}$ and Connie R. Bezzina, PhD ${ }^{1,2}$

\footnotetext{
${ }^{1}$ Department of Clinical and Experimental Cardiology, Heart Centre, Amsterdam Cardiovascular Sciences, Amsterdam UMC, University of Amsterdam, Amsterdam, Netherlands; ${ }^{2}$ Member of the European Reference Network for rare, low prevalence and/or complex diseases of the heart: ERN GUARD-Heart, Amsterdam, Netherlands; ${ }^{3}$ Department of Medicine, Cardiovascular Genetics Center, Montreal Heart Institute and Faculty of Medicine, Université de Montréal, Montreal, QC, Canada; ${ }^{4}$ Université de Nantes, CHU Nantes, CNRS, INSERM, I'institut du thorax, Nantes, France; ${ }^{5}$ Department of Cardiology, The Heart Centre, Copenhagen University Hospital, Rigshospitalet, Copenhagen, Denmark; ${ }^{6}$ Department of Clinical Genetics, Amsterdam UMC, University of Amsterdam, Amsterdam, Netherlands; ${ }^{7}$ National Heart and Lung Institute, Imperial College London, London, United Kingdom; ${ }^{8}$ Cardiovascular Research Centre, Royal Brompton and Harefield NHS Foundation Trust, London, United Kingdom; ${ }^{9}$ MRC London Institute of Medical Sciences, Imperial College London, London, United Kingdom; ${ }^{10}$ Cardiomyopathy Unit, Careggi University Hospital, Florence, Italy; ${ }^{11}$ Department of Experimental and Clinical Medicine, University of Florence, Florence, Italy; ${ }^{12}$ Arrhythmia Section, Cardiology Department, Hospital Clínic, Universitat de Barcelona, Barcelona, Spain; ${ }^{13}$ IDIBAPS, Institut d'Investigació August Pi i Sunyer (IDIBAPS), Barcelona, Spain; ${ }^{14}$ Centro de Investigación Biomédica en Red de Enfermedades Cardiovasculares
} 
(CIBERCV), Madrid, Spain; ${ }^{15} \mathrm{CHU}$ Tours, Service de Cardiologie, Tours, France; ${ }^{16}$ Lankenau Institute for Medical Research, Wynnewood, PA, USA; ${ }^{17}$ Department of Internal Medicine I, LMU Klinikum, Klinikum der Ludwig Maximilians Universität München, München, Germany; ${ }^{18}$ Department of Cardiovascular Medicine, Division of Heart Rhythm Services, Mayo Clinic, Rochester, MN, USA; ${ }^{19}$ Department of Human Genetics, University Hospitals Leuven, Leuven, Belgium; ${ }^{20}$ Cardiovascular Genetics Center, University of Girona-IDIBGI, Girona, Spain; ${ }^{21}$ Medical Science Department, School of Medicine, University of Girona, Girona, Spain; ${ }^{22}$ Biochemistry and Molecular Genetics Department, Hospital Clinic, University of Barcelona-IDIBAPS, Barcelona, Spain; ${ }^{23}$ Istituto Auxologico Italiano, IRCCS-Center for Cardiac Arrhythmias of Genetic Origin, Milan, Italy; ${ }^{24}$ Department of Cardiology, Ege University School of Medicine, Izmir, Turkey; ${ }^{25}$ German Centre for Cardiovascular Research (DZHK), Partner Site Munich, Munich Heart Alliance (MHA), Munich, Germany; ${ }^{26}$ Walter Brendel Centre of Experimental Medicine, Ludwig-Maximilians University Munich (LMU), Munich, Germany; ${ }^{27}$ Center for Human Genetics, University Hospitals Leuven, Leuven, Belgium; ${ }^{28}$ Istituto Auxologico Italiano, IRCCS, Department of Cardiovascular, Neural and Metabolic Sciences, San Luca Hospital, Milan, Italy; ${ }^{29}$ Department of Medicine and Surgery, University of Milano-Bicocca, Milan, Italy; ${ }^{30}$ Istituto Auxologico Italiano, IRCCS-Laboratory of Cardiovascular Genetics, Cusano Milanino, MI, Italy; ${ }^{31}$ Heart Rhythm Management Center, Postgraduate program in Cardiac Electrophysiology and Pacing Universitair Ziekenhuis, Brussel-Vrije Universiteit Brussel, ERN Heart Guard Center, Brussels, Belgium; ${ }^{32}$ CNMR Maladies Cardiaques Héréditaires Rares, Hôpital Bichat, Université de Paris, Paris, France; ${ }^{33}$ AP-HP, Service de Cardiologie, Hôpital Bichat, Paris, France; ${ }^{34}$ Institute for Genetics of Heart Diseases, University Hospital Münster, Münster, Germany; ${ }^{35}$ Cardiac Arrhythmia Service and Cardiovascular Research Center, Massachusetts General Hospital, Boston, MA, USA; ${ }^{36}$ Cardiovascular Disease Initiative, Broad Institute of Harvard and Massachusetts Institute of Technology, Cambridge, MA, USA; ${ }^{37}$ Cardiogenetic Laboratory, Inherited Cardiac Disease Unit, University Hospital Virgen de la Arrixaca-IMIB, Murcia, Spain; ${ }^{38}$ Division of Cardiology, University of Torino, Department of Medical Sciences, "Città della Salute e della Scienza" Hospital, Torino, Italy; ${ }^{39}$ Department of Pediatrics, Kagoshima City Hospital, Kagoshima, Japan; ${ }^{40}$ Center for Epidemiologic Research in Asia, Shiga University of Medical Science, Seta-Tsukinowa-cho, Otsu, Japan; ${ }^{41}$ Omics Research Center, National Cerebral and Cardiovascular Center, Suita, Osaka, Japan; ${ }^{42}$ Division of Patient Safety, Hiroshima University Hospital, Minami-ku, Hiroshima, Japan; ${ }^{43}$ Department of Cardiovascular Medicine, Gunma University Graduate School of Medicine, Maebashi, Gunma, Japan; ${ }^{44}$ Laboratory of Experimental Cardiology, Department of Biomedical Sciences, University of Copenhagen, Copenhagen, Denmark; ${ }^{45}$ Department of Molecular Physiology, Nagasaki University Graduate School of Biomedical Sciences, Nagasaki, Japan; ${ }^{46}$ Department of Clinical Genetics, Maastricht University Medical Center+, Maastricht, Netherlands; ${ }^{47}$ Department of Medicine and Robarts Research Institute, Schulich School of Medicine and Dentistry, Western University, London, ON, Canada; ${ }^{48}$ Center of Medical Genetics, Faculty of Medicine and Health Sciences, University of Antwerp and Antwerp University Hospital, Antwerp, Belgium; ${ }^{49}$ Department of Clinical Genetics and Pathology, Lund University, Lund, Sweden; ${ }^{50}$ Department of Cardiovascular Medicine, Kyoto University Graduate School of Medicine, Kyoto, Japan; ${ }^{51} \mathrm{CHU}$ Brest, Service de Cardiologie, Brest, France; ${ }^{52}$ Univ Rennes, CHU Rennes, INSERM, Rennes, France; ${ }^{53}$ Molecular Cardiology, ICS Maugeri, IRCCS and Department of Molecular Medicine, University of Pavia, Pavia, Italy; ${ }^{54}$ Centre for Cardiovascular Genetics, Department of Public Health and Clinical Medicine, Umeå University, Umeå, Sweden; ${ }^{55}$ Department of Cardiovascular Medicine, Nihon University, Tokyo, Japan; ${ }^{56}$ Molecular and Clinical Sciences Research Institute, St. George's, University of London, London, United Kingdom; ${ }^{57}$ Cardiology Clinical Academic Group, St. George's University Hospitals' NHS Foundation Trust, London, United Kingdom; ${ }^{58}$ Department of Medicine, University Medical Center Mannheim, Mannheim, Germany; ${ }^{59}$ German Center for Cardiovascular Research (DZHK), Partner Site Heidelberg/Mannheim, Mannheim, Germany; ${ }^{60}$ Hôpital Cardiologique du Haut-Lévêque, LIRYC Institute, Université Bordeaux, Bordeaux, France; ${ }^{61}$ Arrhythmias Unit, Hospital Sant Joan de Déu, University of Barcelona, Barcelona, Spain; ${ }^{62}$ Medical Genetics, San Luigi Univesity Hospital, Orbassano, TO, Italy; ${ }^{63}$ Department of Cardiovascular Medicine, National Cerebral and Cardiovascular Center, Suita, Japan; ${ }^{64}$ Department of Medicine, Vanderbilt University Medical Center, Nashville, TN, USA; ${ }^{65}$ Department of Cardiology and Angiology I, Heart Center University of Freiburg, Medical Faculty, Freiburg, Germany; ${ }^{66}$ Department of Pediatrics, National Hospital Organization Kagoshima Medical Center, Kagoshima, Japan; ${ }^{67}$ Department of Cardiovascular Medicine, Kanazawa University Graduate School of Medical Sciences, Kanazawa, Japan; ${ }^{68}$ Department of Genetics, University of Groningen, University Medical Center Groningen, Groningen, Netherlands; ${ }^{69}$ Centre for Medical Genetics, research group Reproduction and Genetics, research cluster Reproduction, Genetics and Regenerative Medicine, Vrije Universiteit Brussel (VUB), Universitair Ziekenhuis Brussel (UZ Brussel), Brussels, Belgium; ${ }^{70}$ Department of Physiology, University of Auckland, Auckland, New Zealand; ${ }^{71}$ Department of Cardiology, CARIM, Maastricht University Medical Center+, Maastricht, Netherlands; ${ }^{72}$ Department of Cardiology, Clinical Sciences, Lund University, Lund, Sweden; ${ }^{73}$ Department of Cardiology, Inselspital, Bern University Hospital, University of Bern, Bern, Switzerland; ${ }^{74}$ Department of Biomedical Informatics, Vanderbilt University Medical Center, Nashville, TN, USA; ${ }^{75}$ Department of Pharmacology, Vanderbilt University Medical Center, Nashville, TN, USA; ${ }^{76}$ Section of Cardiac Electrophysiology, Division of Cardiology, Department of Medicine, Western University, London, ON, Canada; ${ }^{77}$ Cardiac Inherited Disease Group, Starship Childrens Hospital, Auckland, New Zealand; ${ }^{78}$ Department of Forensic Medicine, Faculty of Medical Sciences, University of Copenhagen, Copenhagen, Denmark; ${ }_{79}$ Department of Cardiology, University of Groningen, University Medical Center Groningen, Groningen, Netherlands; ${ }^{80}$ Department of Biomedical Sciences, University of Copenhagen, Copenhagen, Denmark; ${ }^{81}$ Barts Heart Centre, Barts Health NHS Trust \& Institute of Cardiovascular Science, University College London, London, United Kingdom; ${ }^{22}$ Department of Clinical Sciences, Paediatrics, Umeå University, Umeå, Sweden; ${ }^{83}$ Department of Cardiology, University of Antwerp, Antwerp University Hospital, Antwerp, Belgium; ${ }^{84}$ Heart Rhythm Management Center, UZ Brussel-VUB, Brussels, Belgium; ${ }^{85}$ Department of Cardiovascular Diseases, University Hospitals Leuven, Leuven, Belgium; ${ }^{86}$ Medical Genetics Department, Clinical and Biological Sciences, Univestity of Torino, Orbassano, TO, Italy; ${ }^{87}$ Hospital Trueta, CiberCV, University of Girona, IDIBGI, Girona, Spain; ${ }^{88}$ Cardiovascular Institute, Hospital Clinic, University of Barcelona, Barcelona, Spain; ${ }^{89}$ Inherited Cardiac Disease Unit, Cardiology Department, University Hospital Virgen de la Arrixaca, Murcia, Spain; ${ }^{90}$ INSERM, Sorbonne University, UMRS 1166, Institute of Cardiometabolism and Nutrition (ICAN), Paris, France; ${ }^{11}$ Department of Cardiovascular Medicine, Nippon Medical School, Tokyo, Japan; ${ }^{22}$ Department of Bioscience and Genetics, National Cerebral and Cardiovascular Center, Suita, Japan; ${ }^{104}$ Université de Nantes, CNRS, INSERM, l'institut du thorax, Nantes, France 


\section{Nantes Referral Center for inherited cardiac arrhythmia}

Alain Al ArnaoutMD, PhD ${ }^{93}$, Mathieu AmelotMD, PhD ${ }^{94}$, Frédéric AnselmeMD, PhD ${ }^{95}$, Olivier BillonMD, PhD ${ }^{96}$, Pascal DefayeMD, PhD ${ }^{97}$, Jean-Marc DupuisMD, PhD $^{98}$, Laurence JeselMD, PhD ${ }^{99}$, Gabriel LaurentMD, PhD ${ }^{100}$, Philippe MauryMD, PhD ${ }^{101}$, Jean-Luc PasquieMD, PhD ${ }^{102}$ and Francois WiartMD, PhD ${ }^{103}$

\footnotetext{
${ }^{93}$ Service de Cardiologie, GH La Rochelle, La Rochelle, France; ${ }^{94}$ Cardiology Department, CH de Le Mans, Le Mans, France; ${ }^{95}$ Cardiology Department, CHU de Rouen, Rouen, France; ${ }^{96} \mathrm{Chd}$ Les Oudairies, La Roche Sur Yon, France; ${ }^{97} \mathrm{CHU}$ Grenoble, Service de Cardiologie, Grenoble, France; ${ }^{98}$ Service de Cardiologie, CHU d'Angers, Angers, France; ${ }^{99}$ Service de Cardiologie, CHRU de Strasbourg, Strasbourg, France; ${ }^{100}$ Department of Cardiology, University Hospital, Dijon, France; ${ }^{101} \mathrm{CHU}$ Toulouse, Service de Cardiologie, Toulouse, France; ${ }^{102}$ Department of Cardiology, Arnaud de Villeneuve Hospital, Montpellier University Hospital Center, Montpellier, France; ${ }^{103}$ Service de Cardiologie, $\mathrm{CHU}$ de La Réunion, Saint-Denis, Réunion.
} 